\title{
MOLECULAR PHYLOGENETIC ANALYSIS OF MONASCUS FUNGI BASED ON INTERNAL TRANSCRIBED SPACER REGION
}

\author{
N. SUHARNA ${ }^{1}$, Y. KIKUCHI ${ }^{2,3}$ and T. FUKATSU ${ }^{3}$ \\ 'Research Center for Biology, Indonesian Institute of Sciences, Bogor, Indonesia \\ ${ }^{2}$ Natural History Laboratory, Faculty of Science, Ibaraki University, Mito 310-8512, Japan \\ ${ }^{3}$ National Institute of Biological Functions and Resources, Advanced Industrial Science and \\ Technology (AIST), Tsukuba 305-8566, Japan
}

\begin{abstract}
A molecular analysis of internal transcribed spacer region has been carried out to reveal the relationship among 16 strains of Monascus spp. A primer set comprised primer ITS1 and ITS4 was used to amplify this region in which they were cloned and scqucnccd. We also compared the sequence result with M. purpureus AF458473, M.ruber AF458470, M. kaoliang AF451859, M. araneous AF458471 and M. pilosus AF451856 and one outgroup species Thermoascus crustaceus U18353.

The result showed that 16 Monascus spp. were divided into two large clades while M. ruber AF458470 was basically separated from all those Monascus. One of the two large clades included the seven $M$. purpureus strains, $M$. purpureus AF458473, M. araneosus AF458471 and M. kaoliang AF451859. Another large cladc included the six Monascus sp. strains which typically have whitish colonies, the three M. ruber strains and M.pilosus AF451856.

However, even outstanding morphological differences possessed by several white Monascus and one whitish $M$. purpureus strain, all Monascus strains were suggested to be very closely related with similarity $>99 \%$ almost $100 \%$. Although this ITS analysis could not discriminate cultural and morphological differentiation of Monascus strains studied, yet there is still little genetic variation within these strains.
\end{abstract}

Key words : Molecular genetics/Monascus spp./fungi

\section{INTRODUCTION}

Hawksworth and Pitt (1983) cited that Monascus species (Monascaceae) are important for producing Asian fermented foods particularly red rice (ang-kak), rice wine and kaoliang brandy, soy bean cheese and food colorants; for their antibacterial properties; production of mycotoxin; and a major component of silage mycofloras. Lakrodi et al. (200) cited that one of these world wide distributed fungi, $M$. purpureus is known as the red rice fungus that has been used for over a thousand years by the Chinese as a traditional herbal medicine. However, this fungus was firstly isolated from Chinese red rice (ang-kak) in Java.

In Indonesia, we isolated and collected M. purpureus from ang-kak in Java and Sumatra and from other several Monascus species isolated from deteriorated invertebrate specimens. Based on morphological observation on growth in agar media, we found some interesting not ordinary properties in $M$. purpureus such as two $M$. purpureus isolates one of which has unique character such as bigger ascomata and the other one has white colony. While the other four Monascus 
isolates have very remarkable properties such as they resist ethanol at very extreme concentration and their morphological characters are also unique. So, it is of interest to know the genetic relationship rather than morphological relationship among those isolates within Monascus species. Actually, DNA sequence analysis based on the D1/D2 regions of LSU rRNA genes of Monascus species have been conducted by Park and Jong (2003). They suggested that M. lunispora, M. floridanus, and M pallens were separated in different clades. However, five Monascus species such as M pilosus, M. purpureus, M. ruber, M. eremophilus and M. sanguineus were reflected in monophyletic relationship (Park and Jong 2003). These five species were definitely different species based on morphological characters on agar media by Hawksworth and Pitt (1983) (M. pilosus, M. purpureus, M. ruber), Hocking and Pitt (1988) (M eremophilus) and Cannon et al. (1995) (M sanguineus).

As Park and Jong (2003) have suggested no separation among the five species of Monascus, we intended to do analysis on internal transcribed spacer region of Monascus. This region is known for its various nucleotides sequences so it might be more perspective rather than on LSU rRNA genes. This similar work was not only to reveal genetic diversity of Monascus in Indonesia, but also to understand the relationship among Monascus species and direction of mutation by analysis

Monascus Strains

\section{MATERIALS AND METHODS}

A number of 16 Monascus strains used in this study were isolated and identified by Suharna in 2002 and 2003 (Table 1).

Table 1. Monascus strains used

\begin{tabular}{lcl}
\hline \hline \multicolumn{1}{c}{ Monascus Strain } & Isolation Year & \multicolumn{1}{c}{ Source of Isolation } \\
\hline 1. M. purpureus JMBa & 2002 & Fermented Chinese red rice \\
2. M. purpureus JMS & 2002 & Powdered Medicinal Herb \\
3. M. purpureus NGK-J & 1995 & Fermented Chinese red rice \\
4. M. purpureus PKB1 & 2003 & Fermented Chinese red rice \\
5. M. purpureus PKB5 & 2003 & Fermented Chinese red rice \\
6. M. purpureus PRBa & 2002 & Fermented Chinese red rice \\
7. M. purpureus SRBa & 2002 & Fermented Chinese red rice \\
8. M. ruber CKA1 & 2003 & Acid Liquor of Arenga pinnata \\
9. M. ruber CKA3 & 2003 & Acid Liquor of Arenga pinnata \\
10. M. ruber SKF11 & 2003 & Acid Liquor of Arenga pinnata \\
11. Monascus sp. COEL & 1994 & Invertebrate Specimen \\
12. Monascus sp. KA30.1 & 1994 & Invertebrate Specimen \\
13. Monascus sp. KTB & 1994 & Invertebrate Specimen \\
14. Monascus sp. MM & 1994 & Invertebrate Specimen \\
15. Monascus sp. MYOM & 1994 & Invertebrate Specimen \\
16. Monascus sp. MYOT & 1994 & Invetebrate Specimen \\
\hline
\end{tabular}



Molecular phylogenctic analysis of Monascus fungi - N. Suharna et al.

\section{Cultivation and purification of Monascus strains}

All fungi were cultivated on YM Agar plate and for purification of cultures Water Agar 2\% was used. Incubation was carried out at room temperature $\left(25^{\circ} \mathrm{C}\right)$ for three days. A little amount of mycelial mass of each fungus was picked up using toothpick then transferred into centrifuge tube containing $10 \mathrm{ml}$ of $\mathrm{YM}$ broth medium and incubated at $30^{\circ} \mathrm{C}$ for three days. The mycelial mass of each fungus was then harvested for DNA extraction.

\section{DNA extraction}

The harvested mycelial mass of each fungus was put onto paper to remove excess of medium then subsequently put onto mortar before freezing by pouring liquid nitrogen and grounded by mortar and pestle. The following step of DNA extraction was carried out using QIAamp tissue kit (QIAGEN). The yield of DNA samples was quantified and qualified by both spectrophotometer and gel electrophoresis.

\section{PCR, cloning and sequencing of ITS region}

ITS region was amplified by PCR with specific primer set, ITS1 (5'TCCGTAGGTGAACCTGCGG-3') and ITS4 (5'-TCCTCCGCTTATTGATATGC-3') (White et al. 1990). PCR was conducted using AmpliTaq DNA polymerase (Roche, Basel, Switzerland) and supplemented with buffer system under a temperature profile of $94{ }^{\circ} \mathrm{C}$ for $4 \mathrm{~min}$. followed by 30 cycles of $94^{\circ} \mathrm{C}$ for $30 \mathrm{sec}, 55^{\circ} \mathrm{C}$ for $1 \mathrm{~min}$. and $72{ }^{\circ} \mathrm{C}$ for $1 \mathrm{~min}$. The PCR products were directly cloned with TA-cloning vector pT7Blue (Takara) and Escherichia coli DH5a competent cells (Takara) using ampicillin and X-gal blue-white selection system. To check the length of the inserted DNA fragment, white colonies expected to contain inserted plasmid were directly subjected to PCR using the primers Univl9 (5'-GTTTTCCCAGTCACGACGT-3') and Rev20(5'AGCTATGACCATGATTACGC -3'). When a PCR product of expected size (600 bp) was obtained, two clones of which were cultured overnight in $3 \mathrm{ml}$ of LB medium containing ampicillin, and subjected to plasmid extraction using QIAprep-Spin Miniprep Kit (QIAGEN). The purified plasmids were eluted with 50 ul distilled-water and used for sequencing. Dye terminator-labelled cycle sequencing reaction was conducted with DNA Sequencing Kit FS (Perkin Elmer) and four sequencing primers Univl9 and Rev20 under a temperature profile of 94 ${ }^{\circ} \mathrm{C}$ for $4 \mathrm{~min}$. followed by 30 cycles of $94^{\circ} \mathrm{C}$ for $30 \mathrm{sec}, 50{ }^{\circ} \mathrm{C}$ for $1 \mathrm{~min}$. and $60{ }^{\circ} \mathrm{C}$ for $4 \mathrm{~min}$. The products were analyzed by ABI PRISM 377 DNA sequencer (Perkin Elmer). 


\section{Molecular phylogenetic analysis}

The ITS region sequences determined were subjected to molecular phylogenetic analysis together with those retrieved from the DDBJ nucleotide sequence database. A multiple alignment of the ITS region sequences was generated by the program package Clustal W (Thompson et al. 1994). Phylogenetic trees were constructed by the neighbor-joining method using Clustal W (Thompson et al. 1994). Bootstrap tests (Felsenstein 1981) were performed with 1000 replications.

\section{RESULTS}

Figure 1 shows that amplification by primer set comprised ITS1 and ITS4 were successful for all Monascus strain tested.

The size of amplified DNA products of all Monascus isolates by the primer set was 700 base pair each as shown in Figure 1.

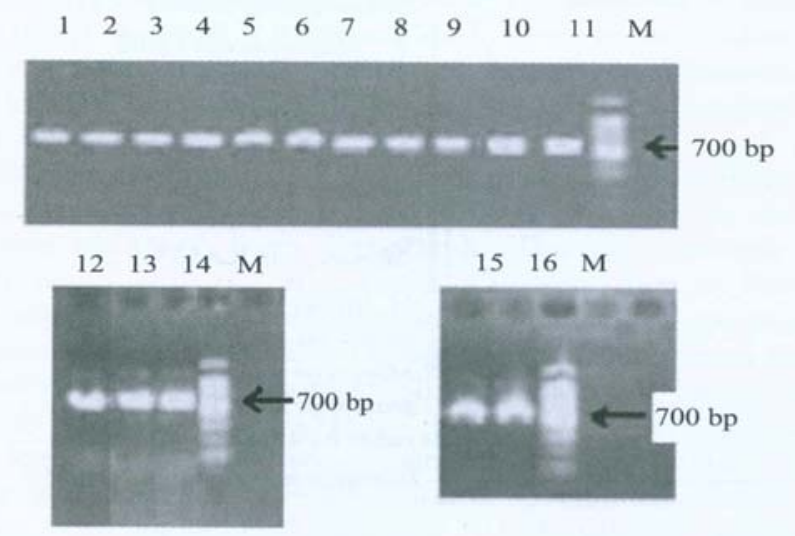

Figure 1. Amplified DNA product of 16 Monascus strains by a primer set comprised primer ITS1 and ITS4. Line: 1. PKB1, 2. JMBa, 3.SKF11, 4.COEL, 5. MM94, 6.JMS, 7.SRBa, 8.KTB, 9.MYOT, 10. MYOM, 11. NGK-J 12.KA30.1, 13.CKA3, 14. CKA 1, 15. PKB5, 16. PRBa.

\section{Molecular phytogeny}

Figure 2 shows that Thermoascus crustaceus U18353 used as outgroup was significantly separated from all Monascus species that integrated in one main clade. This Monascus clade consists of very similar two large clades, while M. ruber AF458470 was basically separated from all those Monascus. One of the two large 
clades includes the seven M. purpureus strains, M. purpureus AF458473, A araneosus AF458471 and M. kaoliang AF451859. Another large clade includes th six Monascus sp. strains which typically have whitish colonies, the three $M$. rube strains and M.pilosus AF451856. The similarities within the seven $M$. purpurei> strains and M. purpureus AF458473, M. araneosus AF458471 and M. kaolian AF451859 are very high more than $99 \%$ or almost $100 \%$. However, M. purpure $\wedge$ PKB5 shows a little more different. The six Monascus sp. strains, the three $M$. rube and M. pilosus AF451856 also showed very high similarity. But lesser difference i shown by Monascus sp. KA30.1.

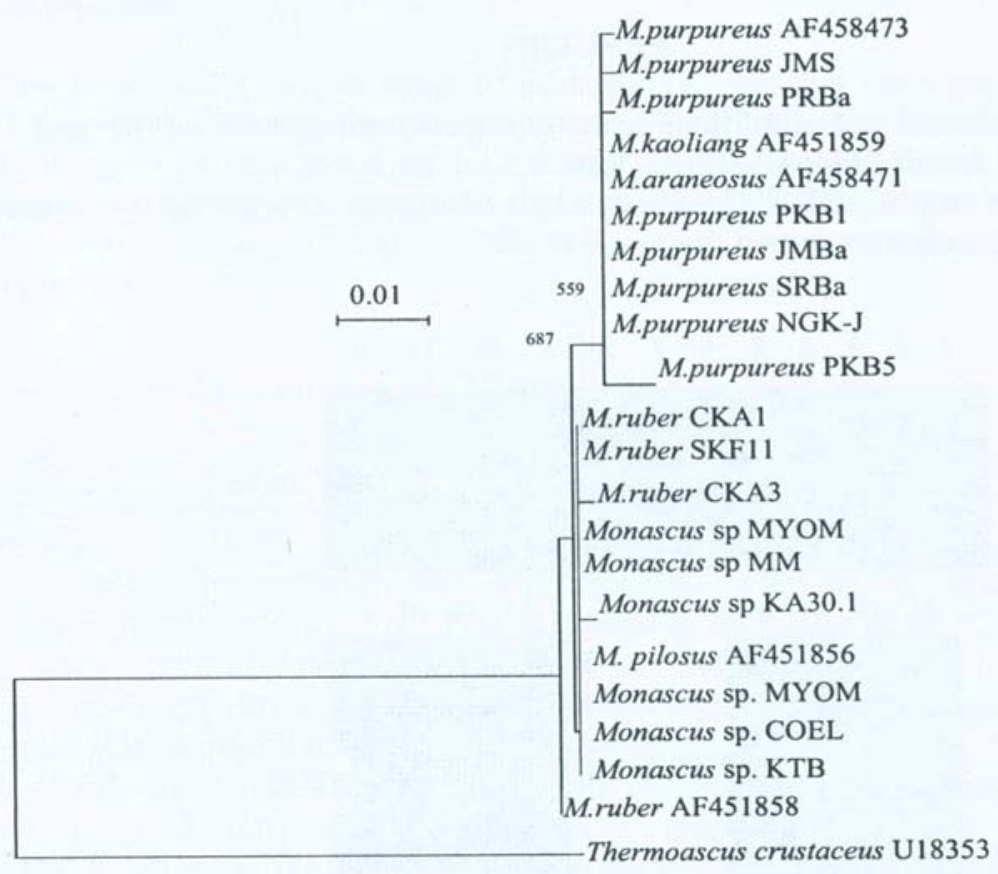

Figure 2. Molecular phylogenetic of 16 Monascus isolates based on internal transcribed spacer sequences. A total of 514 unambiguously aligned nucleotide sites were subjected to neighbor joining analysis.

\section{DISCUSSIONS}

With regard to the level of very high similarity more than 99\% almost nearl; $100 \%$ among all Monascus fungi we analyzed, indicated that there is little mutatioi on ITS region of Monascus species. Concerning genetic diversity it seemed tha there is a diverse mutation tendency as several Monascus strains such a 

M.purpureus PKB1, M.purpureus PRBa, M.purpureus JMS, M.purpureus AF458473, M. rubber CKA1 and Monascus sp. KA30.1 appeared more different though these differences were so slight. Therefore, this phylogenetic analysis revealed very close relationship among Monascus strains so we suggested that all M.purpureus were identical to each other as shown by M.purpureus NGK-J, M.purpureus JMBa, M.purpureus PKB1, M.purpureus PKB5, M.purpureus SRBa, M.purpureus AF458473 and $M$. araneosus AF458471 in the same clade. In another clade, all Indonesian $M$. ruber strains and $M$. pilosus were identical to each other. However, M. ruber AF458470 was separated in another clade. This work also confirmed that M. araneosus AF458471 and $M$. kaoliang AF451859 are the same species with M. purpureus. Previously, Hawkswoth and Pitt (1983) have included M. araneosus and M. kaoliang as M. purpureus synonym based on morphological characteristics. It is also of interest to note that $M$. purpureus SRBa has a uniqueness of its morphological features such as having bigger ascomata (generally 90 um in diameter) than "normal" M. purpureus. The normal M. purpureus has ascomata up to 70 um in diameter. The other white isolates such as Monascus sp. MM, Monascus sp. COEL, Monascus sp. KTB, Monascus sp. MYOM and Monascus sp. MYOT have different morphological characters. These white isolates at least have two different morphological characters compared to $M$. ruber such as shorter ascospores, cleisthothecium wall transparent. Moreover, Monascus sp. MYOM and Monascus sp. MYOT have fusiform aleurispores, while $M$. ruber has no this spore form(Suharna 1999).

Besides D1/D2 regions of LSU rRNA genes, phylogenetic analysis on ITS region was also made by Park and Jong (2003) to know the relationship among Monascus strains accessed from GeneBank. The phylogenetic tree constructed showed similar result with our analysis on ITS sequence of Monascus where $M$. ruber and M. pilosus were very closely related and all $M$. purpureus strains were included together in the same clade. Though it is still suggested that the two clades were very closely related, interestingly, all Indonesian $M$. ruber strains were in the same clade with M. pilosus AF451856. While M. ruber with accession number AF458470 retrieved from DDBJ was separated in another clade. This indicated that three $M$. ruber isolates and six white Monascus isolates showed little difference from M. ruber AF458470, but identical to $M$. pilosus AF451856 except for Monascus sp. KA30.1 and M. ruber CKA3 with genetically little difference.

However, despite of this finding, it is still much surprising in regard with the outstanding morphological differences of several strains as mainly possessed by whitish strains such as Monascus sp. KA30.1, Monascus sp. MYOM, Monascus sp. KTB, Monascus sp. MYOT, Monascus sp. MM, Monascus sp. COEL. Park and Jong (2003) also reported similar result as their comparison of two very distinct species based on morphological observation such as M. ruber and M. pilosus. Therefore, we are in concordance with Park and Jo (2003) that it is needed to reveal in more details about molecular phytogeny to elucidate molecular taxonomy included in the identification of Monascus species. 
Molecular phylogenctic analysis of Monascus fungi-N. Suharna et al.

\section{ACKNOWLEDGMENTS}

This research was funded by the General Exchange Program, Japan Societ The Promotion of Science and conducted in early 2004 at the National Institu Biological Functions and Resources, Advanced Industrial Science and Techno (AIST), Tsukuba 305-8566, Japan.

\section{REFERENCES}

Cannon, P.P. , S.K. Abdullah and B.A. Abbas. 1995. Two new species of Monascus from Iraq, $\backslash$ key to known species of the genus. Mycol. Res. 99: 659-662

Fclsenstcin, J. 1981. Evolutionary Trees from DNA sequences : a maximum likchood approach. J. Evol 17: 368-376

Hawskworth, D.L. \& J.I. Pitt. 1983. A New taxonomy for Monascus based on cultural and micros characters. Aust. J. Bot. 31: $51-61$

Lakrodi, K., C. Chaisrisook, B. Yongsmith and D.Z. Skinner. 2000. RAPD analysis of genetic vari within a collection of Monascus spp. isolated from red rice (ang-kak) and sofu. Mycol. Res (4): 403-408

Park, HG., Jong, S-C. 2003. Molecular characterization of Monascus strains based on the D1/D2 re of LSU rRNA genes. Mycoscicnce 44: 25-32

Thompson, J.D., D.G. Higgins, and J.J. Gibson. 1994. Clustal W: Improving the sensitivity of progrc multiple alignment through sequence weighting, position-specific gap penalties and weight IT choice. Nucleic Acids Res. 22:4673-4680

White, T. J., T. Bruns, S. Lee, and J. W. Taylor. 1990. Amplification and direct sequencing of fi ribosomal RNA genes for phylogcnctics. Pp. 315-322 In: PCR Protocols: A Guide to Method: Applications, eds. Innis, M. A., D. H. Gelfand, J. J. Sninsky, and T. J. White. Academic Press, New York.

Suharna, N. 1999. A study on the fungal occurrences in several animal specimens preserved in alcohc Gakuryoku 5 (3): 139-144 